\title{
L'eSPACE PLIÉ DANS White DE MARIE DARRIEUSSECQ
}

\author{
Judit LIPTÁK-PIKÓ \\ Université de Szeged, Hongrie
}

\begin{abstract}
En): This study attempts to analyse and decipher the writing process in Marie Darrieussecq's 2003 novel White. As in her previous novels, White deals with ghosts and flows of consciousness. Some of the writings of the French philosopher Gilles Deleuze prove to be particularly fruitful while interpreting space as depicted in the novel.
\end{abstract}

Keywords (En): consciousness; fold; smooth; striated; molar; molecular

Mots-clés (Fr) : conscience ; pli ; lisse ; strié ; molaire ; moléculaire

«[...], je dis voyage, mais non pas dans un pays, ou dans un temps. Un voyage dans le sens, dans les mots, dans les sons. On fait un pas de côté, on pénètre un monde parallèle, comme pour quitter la coque humaine. »

Le fantôme est un motif récurrent dans la prose de Marie Darrieussecq : il revient dans Naissance des fantômes (1998), dans Bref séjour chez les vivants (2001) ainsi que dans Tom est mort (2007). Le fantôme peut avoir plusieurs plans d'interprétation. En premier lieu, il peut être conçu comme signe de folie, une sorte de projection du sujet dérangé, ce qui peut entraîner une interprétation psychanalytique lacanienne ou kristevienne de cette figure. En second lieu, le motif du fantôme est sans doute l'élément clé de l'intrigue dans Naissance des fantômes, la prétendue «case vide » selon la rhétorique structuraliste. En troisième lieu, le fantôme est l'allégorie même de la disposition auctoriale, assumée par Marie Darrieussecq : «Écrire, c'est donner voix aux fantômes. » (DARRIEUSSECQ, 2001). Dans cette étude, à travers l'analyse du roman intitulé White, nous proposerons un quatrième plan d'interprétation où le fantôme sera une métaphore ludique des flux et plis de conscience du cerveau.

White (2003) raconte l'histoire d'une équipe scientifique qui part pour le pôle Sud en vue de construire une base européenne permanente au cœur du continent antarctique. La mission s'appelle Projet White, d'où le titre du roman. L'équipe comprend des chercheurs, des glaciologues, un cuisinier, un intendant, un chauffagiste islandais, Peter Tomson, et une standardiste, Edmée Blanco, le seul membre féminin de la compagnie, sa tâche consistant à assurer la communication entre la base et le reste du monde. L'équipe est plus tard rejointe par des ouvriers et 
un astrophysicien ; ce dernier cherche des morceaux de météorites dans la glace ${ }^{1}$. Les événements sont situés dans un futur très proche où les premiers astronautes atterrissent sur Mars et une technologie avancée de télécommunication est en usage sous forme d'hologrammes. Pourtant, en plus d'être l'histoire d'une recherche scientifique, White est avant tout un livre sur les perceptions, les plis de l'espace mental ou la monade (dans le sens leibnizien du mot) d'Edmée et de Peter, le tout englobé dans l'espace antarctique.

Le roman se divise en trois parties dont la première relate, sous la forme de deux volets en alternance, comment l'équipe scientifique accoste la banquise australe : une partie du récit se déroulant dans un bateau, l'autre dans un avion. La deuxième partie relate le séjour dans le campement des scientifiques. Enfin, dans la dernière partie, Peter et Edmée tombent amoureux et la réalisation de leurs désirs sera la cause de l'échec de la mission : Peter, retardé dans le compartiment d'Edmée, n'aura pas assez de temps pour réparer une défaillance du système de chauffage, ainsi l'équipe doit être sauvée en catastrophe. Le roman finit par la naissance d'une nouvelle vie sous le cœur d'Edmée.

White propose aux lecteurs plusieurs portraits : celui d'Edmée et de Peter, et à travers leur portrait conjoint, celui de l'Antarctique, survolé par le «nous » étrange des fantômes ${ }^{2}$. Dans le climat extrême de ce continent, le temps semble «absorbé là, sur place» (DARRIEUSSECQ, 2003: 72), tandis que l'espace lui-même est «ravagé de vide» (DARRIEUSSECQ, 2003: 90). Ce milieu se montre particulièrement propice à l'observation des rêves «fondant aux plis du cerveau » (DARRIEUSSECQ, 2003: 20), au dépliage «des draps de la nostalgie» (DARRIEUSSECQ, 2003 : 142) et où, « dans le silence, dans le blanc, le vide se peuple aussi » (DARRIEUSSECQ, 2003 : 128). La surface du pôle Sud est énorme, elle est plus étendue que celle du continent européen. À cause de son altitude qui s'élève de 2853 mètres jusqu'à 6100 mètres, la respiration est difficile. La température moyenne se situe entre $-20^{\circ}$ et $-60^{\circ}$. À la seule exception des phoques, des manchots, de quelques plantes et des chercheurs, le continent est inhabité. Il en découle qu'à part les journaux et témoignages rédigés par les explorateurs et chercheurs qui y vivent temporairement, on ne peut pas parler de littérature du pôle Sud. Les fantômes des explorateurs qui se sont approchés du pôle, notamment l'Irlandais Ernest Schackleton en 1908, le Norvégien Roald Amundsen en 1911 et le Britannique Robert Scott en 1912 constituent des figures centrales de l'imaginaire antarctique.

\footnotetext{
${ }^{1}$ On pourrait facilement prendre le personnage de l'astrophysicien pour l'avatar du mari de Marie Darrieussecq, qui est lui-même astrophysicien et qui conduit des recherches au pôle Sud. L'écrivaine s'est ouvertement inspirée des expériences de son mari lors de l'écriture de White.

${ }^{2}$ Un jeu énigmatique impliquant les pronoms du singulier (il, elle), du pluriel (nous, ils) et même l'infinitif invite le lecteur à faire très attention au texte. Le jeu avec les pronoms est une démarche sur laquelle Marie Darrieussecq revient souvent, elle s'en est déjà servi dans Mal de mer (P.O.L, 1999).
} 


\section{La mer, archétype de l'espace lisse}

C'est vers ce continent monochrome que se dirige l'équipage du navire ballotté par la houle. La description du mouvement du bateau domine la narration, tout se subordonne aux lois de la mer :

Enfermés dans un Tupperware secoué dans tous les sens. Une étrange lumière grise, la lumière de la mer, en alternance avec du blanc, machine à laver aux hublots. (DARRIEUSSECQ, $2003: 22$ )

La houle insensiblement change d'angle. Corps se balancent de façon incongrue, déroutés, de biais, comme les crabes. [...] La mer n'a plus d'encoches ni de rayures mais une sorte d'ampleur lisse qui enfle puis se rétracte. (DARRIEUSSECQ, 2003 : 27)

L'équipage du bateau retrace le chemin des premiers explorateurs de l'Antarctique dans cette ampleur lisse avec la même intention d'occupation ou, peut-on dire, de territorialisation ou de sédentarisation. La mer, tout comme le ciel ou le désert sont des espaces essentiellement lisses, mis en contraste avec l'espace strié de Gilles Deleuze et de Félix Guattari qui développent cette idée dans le plateau 14, intitulé «Le lisse et le strié » de leur ouvrage Mille plateaux (DELEUZE et GUATTARI, 1980 : 592-593, 597-602). Le trajet des explorateurs, tout comme celui des chercheurs, relie les points dominants : port de débarquement, base scientifique, point central du pôle. Dans le lisse, c'est le contraire, les points sont subordonnés à la ligne tracée par exemple par une population de manchots, dont le mouvement vectoriel est similaire à celui des nomades. L'espace des explorateurs et des chercheurs est avant tout métrique : ils mesurent leur position soit à l'aide d'un sextant, soit à l'aide du GPS. C'est la mer, archétype de tout espace lisse, qui a été confrontée la première à la volonté de striage des marins portugais explorateurs. À la suite du développement de la navigation hautière, la mer est devenue comme quadrillée par la carte, elle-même définie par les lignes de longitude et de latitude. À la méthode de navigation basée sur la position relative du bateau par rapport aux étoiles se substitue une navigation métrique, définie par les degrés et les points. Le dimensionnel a pris le dessus sur le directionnel. Mais il ne faut pas croire que l'existence de ces deux types d'espaces soit séparée, bien au contraire, ils n'existent que par leurs mélanges. Le lisse et le strié sont deux mouvements non symétriques : l'un strie le lisse tandis que l'autre redonne du lisse à partir du strié. Pensons par exemple au mouvement des sous-marins dont la circulation au fond des mers déborde le quadrillage classique de la carte. Pour élargir le champ de leur vision, Deleuze et Guattari projettent le modèle maritime sur la terre ferme, où une application s'impose dans le contexte de la cité, espace strié par excellence à la différence de la mer. Les bidonvilles et les zones défavorisées non répertoriées effacent les striages de la cité de façon rhizomatique et se redistribuent selon une logique lisse. Par contre, dans l'espace strié de la cité, on délimite d'abord une surface, puis «on la répartit suivant des intervalles déterminés » (DELEUZE et GUATTARI, $1980:$ 600). Non seulement les espaces, mais les mouvements aussi peuvent se définir comme lisses et striés. Prenons l'exemple du flâneur, nomade des villes, qui parcourt la cité en lisse, changeant la direction de son trajet en fonction 
de ses expérimentations, des forces survenues, tandis que l'employée ne parcourt que la ligne bien définie entre son logement et son lieu de travail. Il est possible donc d'habiter un espace strié en lisse ou, au contraire, habiter un espace lisse en strié, par exemple la mer, les déserts, les steppes ou le pôle Sud. «C'est le mode de spatialisation, la manière d'être dans l'espace qui distingue les voyages.» (DELEUZE et GUATTARI, 1980 : 602). Nous allons voir dans ce qui suit comment le mode de spatialisation d'Edmée et de Peter change au cours de leur séjour au pôle Sud.

\section{Fantômes ou plis de conscience}

Arrivée sur la banquise, l'équipe scientifique doit faire face à l'épreuve du climat antarctique. La première des choses que l'on remarque est l'absence de végétation et l'immense quantité de neige qui recouvre tout. Le blanc de la neige et le blanc du ciel s'emmêlent, éblouissent et aveuglent la vue par leur lumière nue : « c'est du blanc se diluant dans du blanc» (DARRIEUSSECQ, $2003: 88$ ). L'espace n'est clôturé que par du blanc, «La béance ciel-sol se referme, blanc du ciel contre blanc du sol. » (DARRIEUSSECQ, 2003 : 168). L'horizon est absent, la limite du sol et du ciel est incertaine. Malgré les étendues illimitées, une sensation de vide règne sur tout, une certaine « vacuité de bout du monde » (DARRIEUSSECQ, $2003: 83$ ), l'espace est ravagé par le vide. «Bien sûr, c'est un océan. Un océan de vide congelé. » (DARRIEUSSECQ, 2003 : 158). « On est remarquablement loin de tout, de tout point d'origine, à moins qu'on ne considère le Pôle comme le centre de quelque chose, le centre du vide, alors. » (DARRIEUSSECQ, 2003 : 101-102).

Étrangement, au Pôle, nous avons la sensation de voir cinq soleils en même temps. Cet effet, appelé effet de Parry, est dû à la réfraction de la lumière dans les cristaux de glace en suspension. Encore plus déroutant est le phénomène du white out quand le brouillard se lève et le ciel se reflète dans la neige, et donne la sensation que l'espace entre les deux devient fermé, annulé. À cette impression d'espace ravagé par le vide blanc s'ajoute une perception différée du temps. En raison des jours polaires et du soleil qui ne se couche pas («c'est le matin depuis plusieurs jours », «c'est une seule longue journée » (DARRIEUSSECQ, $2003: 54,65$ )), on a la sensation que le temps est devenu marginal, immobile, indéfini. Le mot «journée » lui-même perd son sens. "La nappe de temps s'étale. La nappe de temps pâle, stationnée sur l'Antarctique. Un anticyclone de temps immobile [...]» (DARRIEUSSECQ, 2003: 125). «Le temps s'enroule et l'espace se perd» (DARRIEUSSECQ, 2003 : 66), tout est dévoré par le vide du blanc.

Dans ce milieu austère, il arrive que les gens qui s'y rendent ont l'impression d'entendre des voix ou d'avoir des hallucinations auditives : des cris lointains, une rumeur de klaxons, une sonnerie de cloches, des chuchotements... «Le silence est

\footnotetext{
${ }^{3}$ L'orientation devient très difficile dans un milieu pareil. En 1979, un vol touristique de la compagnie aérienne nationale néo-zélandaise s'est écrasé contre le mont Erebus. À cause du phénomène de white out, le pilote a pris la muraille de glace verticale pour un champ de glace horizontal. Aucun des passagers ni des membres de l'équipage à bord n'a survécu à l'accident. Cf. : https://fr.wikipedia.org/wiki/Vol_901_Air_New_Zealand
} 
si dense qu'on entend froisser ses épaisseurs » (DARRIEUSSECQ, $2013: 102)^{4}$. Qui est-ce qui émet ces voix, ces bruits ? Dans cette vacuité d'espace, des formes, des êtres étranges commencent à apparaître, ils s'appellent eux-mêmes des fantômes. Qui sont ces fantômes plutôt informes ? En de rares occasions, ils apparaissent sous la forme de brume, d'irisation ou de condensation de l'air, de nuage de poussière flottante, de passage de vent, mais la plupart du temps, ils restent flous, diffus. Après l'arrivée de l'équipe au Pôle, la narration est de plus en plus souvent prise en charge par ces «êtres » étranges sous forme de «nous » qui commencent à peupler le silence et le vide régnant sur l'Antarctique. Le plus souvent, ils surgissent dans des moments d'abandon, dans des moments de "porosité de l'absence à soi-même » (DARRIEUSSECQ, 2013: 77) : ils offrent des moments de nostalgie de la vie d'Edmée et de Peter, prennent souvent la forme de rêveries, de fantaisies, de désirs. En effet, ces fantômes sont des pensées nomades qui, dans le silence, peuplent l'espace environnant. Ils prennent en compte toutes sortes d'idées qui surviennent dans le cerveau humain, «la peur des insectes, l'attraction du vide, le goût de la fuite, l'ennui, les maladresses, les hantises et les phobies, et les désirs, les vertiges, les drames, et les trésors qui à la longue s'accumulent ou se défont» (DARRIEUSSECQ, 2003 : 50). Leurs mouvements zigzagants ressemblent à la nature aléatoire de l'apparition des bribes de pensées aux moments d'abandon à soi. White est avant tout un questionnement sur le vide, un roman sur le mode opératoire rhizomatique des pensées et du cerveau aux moments vides ${ }^{5}$. La condition immatérielle des fantômes, leurs mouvements indépendants du temps et de l'espace participent d'un univers dit chaosmotique où « les séries divergentes tracent dans un même monde chaotique des sentiers toujours bifurcants » (DELEUZE, $1988: 11$ ).

$\mathrm{Au}$ fil des pages, nous assistons à des processus de dépliage de perceptions. Nous empruntons la notion à Gilles Deleuze qui dans Le pli. Leibniz et le baroque effectue une lecture critique de La Monadologie de Gottfried Leibniz. Dans l'acception leibnizienne, chaque humain représente une monade qui est elle-même composée d'un nombre infini de plis. Le monde n'existe que sous forme virtuelle qui s'actualise dès qu'un individu, une monade, l'exprime et donne ainsi une représentation du monde (DELEUZE, $1988: 32$ ). Deleuze identifie plusieurs types de perceptions : tandis que les microperceptions ou perceptions moléculaires sont perçues inconsciemment, les perceptions molaires sont conscientes et seules, elles peuvent constituer l'objet d'une verbalisation. Or, tout l'enjeu de l'écriture littéraire réside là : à savoir, comment passer de la perception moléculaire à la perception molaire (DELEUZE, 1988: 116), comment passer de l'état inconscient à la représentation. Autrement dit, comment donner forme à l'informe ou pour citer

\footnotetext{
${ }^{4}$ Il ne serait d'ailleurs pas difficile d'esquisser le paysage auditif ou le soundscape du bateau qui tangue ou celui du Pôle, tellement Marie Darrieussecq emploie des onomatopées et des verbes qui expriment un certain effet de bruit.

5 « Depuis longtemps je travaille sur le thème du vide, et sur deux questions : que fait-on quand on ne fait rien? et où est le centre du monde? Être bloqué des mois au centre de nulle part, environné de blanc, perdu dans un temps et un espace problématiques, était une expérience que je voulais explorer. Cela dirait forcément quelque chose de l'humain. » Entretien réalisé par Amy Concannon et Kerry Sweeney en mars 2004. http://darrieussecq.arizona.edu/fr/entretien-r\%C3\%A9alis\%C3\%A9-paramy-concannon-et-kerry-sweeney-en-mars-2004 [consulté le 10 janvier 2017].
} 
Deleuze : «[...] l'informel n'est pas négation de la forme : il pose la forme comme pliée et n'existant que comme «paysage du mental », dans l'âme ou dans la tête [...] (DELEUZE, 1988 : 49-50). Cette question complexe de l'écriture liée à la conscience apparaît également chez Julia Kristeva dans La révolution du langage poétique où elle développe deux définitions inspirées par la psychanalyse lacanienne, notamment le sémiotique et le symbolique. Kristeva entend par sémiotique toute chose préverbale, hétérogène, antérieure à la nomination, ce qui ne se laisse pas ramener au sens et à la signification et dont participe considérablement le langage poétique. Le symbolique, lui, se rattache à la fonction de la signifiance, de la représentation. (KRISTEVA, 1974 : 149-172). De même, Nathalie Sarraute, dans son essai intitulé L'ère du soupçon, donne le nom de tropisme aux flux de conscience fugaces, brefs, intenses et inexpliqués : «[...] l'expression spontanée d'impressions très vives, et leur forme était aussi spontanée et naturelle que les impressions auxquelles elle donnait vie. [...] $\mathrm{Ce}$ sont des mouvements indéfinissables, qui glissent très rapidement aux limites de notre conscience $[\ldots]$ » (SARRAUTE, $1956: 8$ ). Ce qui est le moléculaire pour Deleuze, le sémiotique pour Kristeva ou le tropisme pour Sarraute, c'est le fantôme pour Marie Darrieussecq.

\section{Défaire les plis de l'espace antarctique}

Edmée et Peter entrent en rapport avec l'Antarctique, ils deviennent petit à petit comme absorbés par cet espace polaire avec lequel ils forment un composé de sensations que l'on pourrait appeler le Pôle.

Peter voudrait absorber tout le paysage. Il tourne sur lui-même. Englober, d'un coup, comprendre : tout le paysage. L'air, le soleil, le sol. Ce paysage habité par eux seuls, ce non-lieu, ce non-sens formidable, air soleil sol. Habité par eux seuls. Crevasses et craquements, et le désir de tout prendre, infini, juste en se tenant là, debout. Puisque arpenter est impossible. Puisque recenser, cadastrer, détailler est impossible. Devenir poreux, se laisser rapter par l'espace qui creuse ici un point immobile, et ne se met en branle, événement, cahots de machine, qu'avec ses pas à lui, Peter. (DARRIEUSSECQ, $2003: 154-155$ )

N'être plus qu'un souffle, flux et reflux, passage de l'air... être poreuse au monde, posée là, n'importe où, sur des braises, au fond d'un lac, dans une tente surchauffée, et ne penser à rien, même pas penser qu'on pense à rien - elle savait y faire avant. (DARRIEUSSECQ, 2003 : 76-77)

Sous la plume de Darrieussecq, le Pôle Sud devient un être de sensation, elle lui crée des yeux, «le paysage voit » (DELEUZE, $1991: 159$ ). Des affects et des percepts naissent de ce rapport mutuel entre Edmée, Peter et le paysage qui rendent « sensibles les forces insensibles qui peuplent le monde, et qui nous affectent, nous font devenir » (DELEUZE, 1991 : 172). Une définition que Deleuze donne au percept et à l'affect dans Pourparlers : «Les percepts ne sont pas des perceptions, ce sont des paquets de sensations et de relations qui survivent à celui qui les éprouve. Les affects ne sont pas des sentiments, ce sont des devenirs qui débordent celui qui passe par eux (il devient autre). »(DELEUZE 1990 : 187). Edmée et Peter existent parce qu'ils passent dans le paysage et «font eux-mêmes partie du composé de sensations » (DELEUZE 1990 : 159-160). Pareillement à Achab qui, à travers son rapport avec Moby Dick, forme un composé de sensations qui s'appelle l'Océan. 
«On n'est pas dans le monde, on devient avec le monde, on devient en le contemplant. » (DELEUZE 1990 : 160) - écrit Deleuze dans le chapitre «Affect, percept et concept » de Qu'est-ce que la philosophie ?. « Est-ce qu'on peut appeler ça un paysage ? » (DARRIEUSSECQ, 2003 : 35) - demande le narrateur dans White. Oui, un paysage où Edmée et Peter ont un devenir-paysage et le paysage un devenirEdmée et devenir-Peter.

Dans le vide, Edmée et Peter deviennent poreux aux forces et aux intensités de l'espace polaire qui sont communiquées par les fantômes ${ }^{6}$. Parallèlement, leur façon d'exister dans l'espace change : ils commencent à habiter en lisse l'espace strié initial de la mission scientifique. Leur perception devient haptique plutôt qu'optique, sensible aux heccéités du cerveau. Un espace imaginaire et rhizomatique, tissé pêle-mêle par des lignes de pensée fuyantes, émerge et se substitue lentement à l'espace métrique et cartographié de la base scientifique. Le mouvement déterritorialisant de cette machine de guerre à deux fait échouer finalement l'appareil d'État de la mission: Edmée et Peter font l'amour et ne remarquent pas le vacarme produit par l'alarme qui signale une faille dans le système de chauffage. À cause de la perte de temps, l'erreur ne peut plus être rectifiée, les membres de l'équipe scientifique doivent être évacués dans les plus brefs délais et la mission échoue.

Malgré l'austérité du milieu antarctique, c'est un espace mental complexe et mouvementé qui apparaît dans White. La multitude des plis remplit le vide blanc de percepts et d'affects. En héritière des auteurs énumérés ci-dessus (Kisteva, Deleuze, Sarraute, etc.), Darrieussecq explore dans la plupart de ses livres la dimension des plis de conscience, peu importe l'arrière-plan de l'intrigue: que ce soit en Antarctique, dans une ville côtière imaginée (Naissance des fantômes, Mal de mer), en Australie (Tom est mort), dans le pays Yuoangui (Le Pays) ou sans lieu précis (Bref séjour chez les vivants). En guise de conclusion, citons les propos de l'écrivaine :

Le lieu d'où j'écris a la forme d'un pli, ou d'un littoral : là où la mer arrive sur la terre, dans ce creux-là, dans ce rouleau. Juste avant que la vague casse, dans le vide bref, renouvelé, sonore, j'aime penser que j'écris de là. Cet espace, je le trouve partout. Même en montagne, dans les rimayes, entre la neige et la pierre. Je le trouve au bord des fleuves, aux lisières des forêts, aux angles des trottoirs, et chez moi. Ce n'est pas le lieu d'un vertige, ni d'une chute ni d'un tourbillon. C'est un lieu mobile mais stable, un repère en mouvement. (DARRIEUSSECQ, 2013)

\section{BIBLIOGRAPHIE}

DARRIEUSSECQ Marie (2003), White, Paris, Éditions Gallimard.

DARRIEUSSECQ Marie (2013), J'écris d'un monde tellurique, fleuvien..., http://www.liberation.fr/planete/2013/08/13/j-ecris-d-un-monde-telluriquefleuvien_924633

DeleuZE Gilles ; GUATTARI Félix (1980), Mille plateaux, Paris, Éditions de Minuit.

\footnotetext{
${ }^{6}$ « C'est pourquoi, ce qui occupe l'espace lisse, ce sont les intensités, les vents et les bruits, les forces et les qualités tactiles et sonores, comme dans le désert, la steppe ou les glaces. Craquement de la glace et chant des sables. » (Deleuze, $1980: 598)$.
} 
DeLEUZE Gilles (1988), Le pli. Leibniz et le baroque, Paris, Éditions de Minuit.

Deleuze Gilles (1990), Pourparlers, Paris, Éditions de Minuit.

DELEUZE Gilles (1991), Qu'est-ce que la philosophie ?, Paris, Éditions de Minuit.

KRISTEVA Julia (1974), La révolution du langage poétique, Paris, Éditions du Seuil.

LE ClezIO Jean-Marie Gustave (2003), Critique de White, paru dans Le Point, http://www.pol-editeur.com/index.php?spec=livre \&ISBN=2-86744-962-6

SARRAUTE Nathalie (1956), L'ère du soupçon, Paris, Éditions Gallimard.

\section{Entretiens}

Entretien réalisé par Becky Miller en 2001, http://darrieussecq.arizona.edu/fr/entretien-r $\% \mathrm{C} 3 \% \mathrm{~A} 9 \mathrm{alis} \% \mathrm{C} 3 \% \mathrm{~A} 9-$ parbecky-miller-et-martha-holmes-en-d\%C3\%A9cembre-2001

Entretien réalisé dans le cadre du séminaire Habiter. L'Encrage en littérature contemporaine organisé par Claire Colard et Zoé Courtois en 2016, https://www.youtube.com/watch?v=290sfuLKKA0\&t=306s 